\title{
Unsteady Magnetohydrodynamic Convective Heat and Mass Transfer Past an Infinite Vertical Plate in a Porous Medium with Thermal Radiation, Heat Generation/Absorption and Chemical Reaction
}

\author{
Stanford Shateyi ${ }^{1}$ and Sandile Motsa ${ }^{2}$ \\ ${ }^{1}$ University of Venda \\ ${ }^{2}$ University of Swaziland \\ ${ }^{1}$ South Africa \\ ${ }^{2}$ Swaziland
}

\section{Introduction}

Convective flows with simultaneous heat and mass transfer under the influence of a magnetic field and chemical reaction arise in many transport processes both naturally and artificially in many branches of science and engineering applications. This phenomenon plays an important role in the chemical industry, power and cooling industry for drying, chemical vapour deposition on surfaces, cooling of nuclear reactors and petroleum industries.

Natural convection flow occurs frequently in nature. It occurs due to temperature differences, as well as due to concentration differences or the combination of these two, for example in atmospheric flows, there exists differences in water concentration and hence the flow is influenced by such concentration difference.

Changes in fluid density gradients may be caused by non-reversible chemical reaction in the system as well as by the differences in molecular weight between values of the reactants and the products. Chemical reactions can be modeled as either homogeneous or heterogeneous processes. This depends on whether they occur at an interface or as a single phase volume reaction. A homogeneous reaction is one that occurs uniformly throughout a given phase. On the other hand, a heterogeneous reaction takes place in a restricted area or within the boundary of a phase. In most cases of chemical reactions, the reaction rate depends on the concentration of the species itself. A reaction is said to be of first order, if the rate of reaction is directly proportional to the concentration itself, (Cussler, 1988). For example, the formation of smog is a first order homogeneous reaction. Consider the emission of nitrogen dioxide from automobiles and other smoke-stacks. This nitrogen dioxide reacts chemically in the atmosphere with unburned hydrocarbons (aided by sunlight) and produces peroxyacetylnitrate, which forms an envelop which is termed photo-chemical smog.

The study of heat and mass transfer with chemical reaction is of great practical importance in many branches of science and engineering. (Das et al., 1994) studied the effects of mass transfer flow past an impulsively started infinite vertical plate with constant heat flux and chemical reaction. (Anjalidevi \& Kandasamy, 1999) studied effects of chemical reaction, heat and mass transfer on laminar flow along a semi-infinite horizontal plate. More recently, intensive studies have been carried out to investigate effects of chemical reaction on different 
flow types (see Seddeek et al. 2007; Salem \& Abd El-Aziz 2008; Mohamed 2009; Ibrahim et al.,2008).

Convection flows in porous media has gained significant attention in recent years because of their importance in engineering applications such as geothermal systems, solid matrix heat exchangers, thermal insulations, oil extraction and store of nuclear waste materials. These can also be applied to underground coal gasification, ground water hydrology, wall cooled catalytic reactors, energy efficient drying processes and natural convection in earth's crust. Detailed reviews of flow through and past porous media can be found in (Nield and Benjan, 1999). (Abel et al., 2001) studied the two-dimensional boundary layer problem on mixed convection of an incompressible visco-elastic fluid immersed in a porous medium over a stretching sheet. (Ali, 2007) analyzed the effect of lateral mass flux on natural convection boundary layer induced by heated vertical plate embedded in a saturated porous medium.

The use of magnetic field that influences heat generation/absorption process in electrically conducting fluid flows has many engineering applications. For example, many metallurgical processes which involve cooling of continuous strips or filaments, which are drawn through a quiescent fluid. The properties of the final product depend to a great extent on the rate of cooling. The rate of cooling and therefore, the desired properties of the end product can be controlled by the use of electrically conducting fluids and the applications of the magnetic fields (Vajravelu \& Hadjinicalaou 1997). Many works have been reported on flow, heat and mass transfer of electrically conducting fluids over semi-infinite/infinite plates/stretching surfaces in the presence of magnetic field (see for instance Chamkha \& Khaled, 2000; Shateyi et al. 2007, 2010; Makinde \& Sibanda 2008; Bég et al. 2009, Pal \& Talukdar 2010, Makinde \& Aziz 2010).

The study of heat generation or absorption in moving fluids is important in problems dealing with chemical reactions and those concerned with dissociating fluids. Heat generation effects may alter the temperature distribution and this in turn can affect the particle deposition rate in nuclear reactors, electronic chips and semi conductor wafers. Although exact modeling of internal heat generation or absorption is quite difficult, some simple mathematical models can be used to express its general behaviour for most physical situations. Heat generation or absorption can be assumed to be constant, space-dependent or temperature-dependent. (Crepeau \& Clarksean 1997) have used a space-dependent exponentially decaying heat generation or absorption in their study on flow and heat transfer from a vertical plate. Several interesting computational studies of reactive MHD boundary layer flows with heat and mass transfer in the presence of heat generation or absorption have appeared in recent years (see for example Patil \& Kulkarni, 2008; Salem \& El-Aziz, 2008; Samad \& Mohebujjaman, 2009; Mohamed, 2009; Mahdy, 2010 ).

When technological processes take place at higher temperatures thermal radiation heat transfer has become very important and its effects cannot be neglected (Siegel \& Howel, 2001). The effect of radiation on MHD flow, heat and mass transfer become more important industrially. Many processes in engineering areas occur at high temperature and a knowledge of radiation heat transfer becomes a very important for the design of the pertinent equipment. The quality of the final product depends to a great extent on the heat controlling factors, and the knowledge of radiative heat transfer in the system can lead to a desired product with sought qualities. Different researches have been forwarded to analyze the effects of thermal radiation on different flows (Cortell, 2008; Bataller, 2008; Ibrahim et al. 2008; Shateyi, 2008; Shateyi and Motsa, 2009; Aliakba et al., 2009; Shateyi et al., 2010, Hayat, 2010; Cortell, 2010; among other researchers). 
In spite of all the previous studies, the unsteady MHD free convection heat and mass transfer for a heat generation/absorption with radiation absorption in the presence of a reacting species over an infinite permeable plate has received little attention. Hence, the main objective of this chapter is to investigate the effects of thermal radiation, chemical reaction, heat source/sink parameter of an electrically conducting fluid past an infinite vertical porous plate subjected to variable suction. The plate is assumed to be embedded in a uniform porous medium and moves with a constant velocity in the flow direction in the presence of a transverse magnetic field. The governing equations are transformed into a system of nonlinear ordinary differential equations by using suitable similarity transformations. Numerical calculations are carried out using the spectral homotopy analysis method (SHAM). The SHAM is a modified version of the homotopy analysis method (HAM), (Liao, 2003) and the Chebyshev spectral collocation method (Trefethen, 2000). The SHAM has been successfully used by (Motsa et al. 2010a,b). One strength of the SHAM is that it removes restrictions of the HAM such as the requirement for the solution to conform to the so-called rule of solution expression and the rule of coefficient ergodicity. Also, the SHAM inherits strengths of the HAM, for example, it does not depend on the existence of a small parameter in the equation to be solved, it avoids discretization, and the solution obtained is in terms of an auxiliary parameter $\hbar$ which can be conveniently used to determine the convergence rate of the solution.

Graphical results for the velocity, temperature and concentration profiles based on the numerical solutions are presented and discussed. We also discuss the effects of various parameters on the skin-friction coefficient and the rate of heat and mass transfer at the surface.

\section{Mathematical formulation}

We consider an unsteady two-dimensional flow of an incompressible and electrically conducting viscous fluid, along an infinite vertical permeable plate embedded in a porous medium, with thermal radiation, heat generation/absorption and chemical reaction. The $x-$ axis is taken on the infinite plate, and parallel to the free-stream velocity which is vertical and the $y$ - axis is taken normal to the plate. Since the motion is two dimensional and the length of the plate is large enough, all the physical variables are independent of $x$. A magnetic field $B_{0}$ of uniform strength is applied transversely to the direction of the flow. The transversely applied magnetic field and magnetic Reynolds number are assumed to be very small so that the induced magnetic field and the Hall effect are negligible. In this chapter assumption is also made that there is no applied voltage which implies the absence of an electric field. The plate is maintained at constant temperature $T_{w}$ and concentration $C_{w}$, higher than the ambient temperature $T_{\infty}$ and concentration $C_{\infty}$, respectively.

The analysis considers a homogeneous first-order chemical reaction with constant rate $k_{c}$ between the diffusing species and the fluid. The fluid is assumed to have constant properties except that the influence of the density variations with temperature and concentration which are considered only in the body force term. The governing equations for the problem considered in this chapter are based on the balances of mass, linear momentum, energy and concentration species. These equations are as given below:

Continuity equation:

$$
\frac{\partial v}{\partial y}=0
$$


Momentum equation:

$$
\frac{\partial u}{\partial t}+v \frac{\partial u}{\partial y}=v \frac{\partial^{2} u}{\partial y^{2}}+g \beta_{t}\left(T-T_{\infty}\right)+g \beta_{c}\left(C-C_{\infty}\right)-\frac{\sigma B_{0}^{2}}{\rho} u-\frac{v}{K} u-\frac{b}{K} u^{2},
$$

Energy equation:

$$
\frac{\partial T}{\partial t}+v \frac{\partial T}{\partial y}=\alpha \frac{\partial^{2} T}{\partial y^{2}}-\frac{1}{\rho c_{p}} \frac{\partial q_{r}}{\partial y}-\frac{Q_{0}}{\rho c_{p}}\left(T-T_{\infty}\right)+Q_{1}^{*}\left(C-C_{\infty}\right),
$$

Mass diffusion equation:

$$
\frac{\partial C}{\partial t}+v \frac{\partial C}{\partial y}=D \frac{\partial^{2} C}{\partial y^{2}}-k_{c}\left(C-C_{\infty}\right)
$$

Where $x, y$ are the dimensional distance along and perpendicular to the plate, respectively. $u$ and $v$ are the velocity components in the $x, y$ directions respectively, $g$ is the gravitational acceleration, $\rho$ is the fluid density, $\beta_{t}$ and $\beta_{c}$ are the thermal and concentration expansion coefficients respectively, $K$ is the Darcy permeability, $b$ is the emperical constant, $B_{0}$ is the magnetic induction, $T$ is the thermal temperature inside the thermal boundary layer and $C$ is the corresponding concentration, $\sigma$ is the electric conductivity, $\alpha$ is the thermal diffusivity, $c_{p}$ is the specific heat at constant pressure, $D$ is the diffusion coefficient, $q_{r}$ is the heat flux, $Q_{0}$ is the dimensional heat absorption coefficient, $Q_{1}^{*}$ is the coefficient of proportionality of the radiation and $k_{c}$ is the chemical reaction parameter.

The boundary conditions for the velocity, temperature and concentration are:

$$
\begin{aligned}
& u=U_{0}, \quad v=v(t), \quad T=T_{w}, \quad C=C_{w} \text { at } y=0 \\
& u=0, \quad T=T_{\infty}, \quad C=C_{\infty}, \quad \text { as } y \rightarrow \infty .
\end{aligned}
$$

By using the Rosseland diffusion approximation (Hossain et al. 1999) and following (Raptis, 1999) among other researchers, the radiative heat flux, $q_{r}$ is given by

$$
q_{r}=-\frac{4 \sigma^{*}}{3 K_{s}} \frac{\partial T^{4}}{\partial y}
$$

where $\sigma^{*}$ and $K_{s}$ are the Stefan-Boltzman constant and the Roseland mean absorption coefficient, respectively. We assume that the temperature differences within the flow are sufficiently small such that $T^{4}$ may be expressed as a linear function of temperature.

$$
T^{4} \approx 4 T_{\infty}^{3} T-3 T_{\infty}^{4}
$$

Using (7) and (8) in the last term of equation (3) we obtain

$$
\frac{\partial q_{r}}{\partial y}=-\frac{16 \sigma^{*} T_{\infty}^{3}}{3 K} \frac{\partial^{2} T}{\partial y^{2}}
$$

In order to obtain a local similarity solution (in time) of the problem under consideration we introduce a time dependent length scale $\delta$ as

$$
\delta=\delta(t)
$$


A convenient solution of equation (1) in terms of this length scale is considered to be in the following form

$$
v=v(t)=-\frac{v}{\delta} v_{0}
$$

where $v_{0}>0$ is the suction parameter.

These non-linear partial differential equations are then transformed by a similarity transformation into a system of ordinary differential equations given as;

$$
\begin{aligned}
& f^{\prime \prime}+\left(2 \eta+v_{0}\right) f^{\prime}+G r \theta+G c \phi-M f-\frac{1}{D a} f-\frac{R e F s}{D a} f^{2}=0, \\
& \left(\frac{1+R}{P r}\right) \theta^{\prime \prime}+\left(2 \eta+v_{0}\right) \theta^{\prime}-\Omega \theta+Q_{2} \phi=0, \\
& \frac{1}{S c} \phi^{\prime \prime}+\left(2 \eta+v_{0}\right) \phi^{\prime}-\gamma \phi=0,
\end{aligned}
$$

where primes denote differentiation with respect to $\eta$ and $D a=\frac{K}{\delta^{2}}$ is the local Darcy number, $F s=\frac{b}{\delta}$ is the local Forchheimer number, $R e=\frac{U_{0} \delta}{v}$ is the local Reynolds number, $\operatorname{Pr}=\frac{v}{\alpha}$ is the Prandtl number, $\Omega=\frac{Q_{0}}{\rho c_{p}}$ is the heat absorption parameter, $Q_{2}=\frac{Q_{1}^{*}\left(C_{w}-C_{\infty}\right)}{(T-T \infty}$ is the absorption of radiation parameter, $\gamma=\frac{k_{c} \delta^{2}}{v}$ is the chemical reaction parameter, $S c=\frac{v}{D}$ is the Schmidt number, $M=\frac{\sigma \delta^{2} B_{0}^{2}}{v \rho}$ is the magnetic field parameter, $R$ is the thermal radiation parameter, $G r=$ $g \beta_{t}\left(T_{w}-T_{\infty}\right) \delta^{2} / v U_{0}$ is the local Grashof number, $G c=g \beta_{c}\left(C_{w}-C_{\infty}\right) \delta^{2} / U_{0}$ is the modified Grashof number.

The corresponding boundary conditions for $t>0$ are transformed to:

$$
\begin{aligned}
& f=1, \quad \theta=1, \quad \phi=1, \text { at } \eta=0, \\
& f=0, \quad \theta=0, \phi=0 \text { as } \eta \rightarrow \infty .
\end{aligned}
$$

The resultant system of coupled and non-linear ordinary differential equations are then solved by the Spectral homotopy analysis method (SHAM). The effects of various parameters such as local Darcy number, Schmidt number, Hartmann, radiation parameter, reaction rate parameter and Grashof numbers on the velocity, temperature, concentration, skin-friction coefficient, Nusselt number and Sherwood numbers are shown in figures and tables and analyzed in detail.

\section{Solution methods}

To solve the equation system (12) - (14) we first use the Chebyshev pseudospectral method (CPM) to solve equation (14) for $\phi(\eta)$ and use the result in (13) to solve for $\theta(\eta)$ using the Chebyshev pseudospectral method again. The solutions for $\theta(\eta)$ and $\phi(\eta)$ are substituted in (12) to obtain a nonlinear equation in $f(\eta)$. To solve the resulting nonlinear equation we use the spectral homotopy analysis method (SHAM) which is based on a blend of the Chebyshev pseudospectral method with the standard homotopy analysis method (HAM).

Before applying the CPM and SHAM we use the domain truncation approach to approximate the domain of the problem $[0, \infty)$ by the numerical computational domain $[0, L]$, where $L$ is a fixed length that is taken to be sufficiently larger than the thickness of the boundary layer. 
We then transform the domain $[0, L]$ to the domain $[-1,1]$, on which the Chebyshev spectral method can be implemented, using the algebraic mapping

$$
\xi=\frac{2 \eta}{L}-1, \quad \xi \in[-1,1] .
$$

To solve (13) and (14) we use the Chebyshev pseudospectral method and approximate the unknown functions $\theta(\xi)$ and $\phi(\xi)$ as a truncated series of Chebyshev polynomials of the form

$$
\theta(\xi) \approx \sum_{k=0}^{N} \theta_{k} T_{k}\left(\xi_{j}\right), \phi(\xi) \approx \sum_{k=0}^{N} \phi_{k} T_{k}\left(\xi_{j}\right), \quad j=0,1, \ldots, N
$$

where $T_{k}$ is the $k$ th Chebyshev polynomial, and $\xi_{0}, \xi_{1}, \ldots, \xi_{N}$ are Gauss-Lobatto collocation points (see Canuto et al. 1988) defined by

$$
\xi_{j}=\cos \frac{\pi j}{N}, \quad j=0,1, \ldots, N .
$$

and $N+1$ is the number of collocation points. Derivatives of the functions $\theta(\eta)$ and $\phi(\eta)$ at the collocation points are represented as

$$
\frac{d^{r} \theta}{d \eta^{r}}=\sum_{k=0}^{N} \mathbf{D}_{k j}^{r} \theta\left(\xi_{j}\right), \quad \frac{d^{r} \phi}{d \eta^{r}}=\sum_{k=0}^{N} \mathbf{D}_{k j}^{r} \phi\left(\xi_{j}\right)
$$

where $r$ is the order of differentiation and $\mathbf{D}=\frac{2}{L} \mathcal{D}$ with $\mathcal{D}$ being the Chebyshev spectral differentiation matrix (see for example, Canuto et al. 1988; Trefethen 2000) whose entries are defined as

$$
\begin{aligned}
& \mathcal{D}_{j k}=\frac{c_{j}}{c_{k}} \frac{(-1)^{j+k}}{\xi_{j}-\xi_{k}} \quad j \neq k ; j, k=0,1, \ldots, N, \\
& \mathcal{D}_{k k}=-\frac{\xi_{k}}{2\left(1-\xi_{k}^{2}\right)} \quad k=1,2, \ldots, N-1, \\
& \mathcal{D}_{00}=\frac{2 N^{2}+1}{6}=-\mathcal{D}_{N N} .
\end{aligned}
$$

Substituting equations (18) - (21) in (13) yields

$$
\mathbf{C} \Phi=0
$$

subject to the boundary conditions

$$
\phi\left(\xi_{N}\right)=1, \quad \phi\left(\xi_{0}\right)=0,
$$

where

$$
\begin{aligned}
\mathbf{C} & =\frac{1}{S c} \mathbf{D}^{2}+\left(2 \mathbf{j}+v_{0} \mathbf{I}\right) \mathbf{D}-\gamma \mathbf{I}, \\
\boldsymbol{\Phi}_{0} & =\left[\phi\left(\xi_{0}\right), \phi\left(\xi_{1}\right), \ldots, \phi\left(\xi_{N}\right)\right]^{T}, \\
\mathbf{j} & =\operatorname{diag}\left(\left[\eta_{0}, \eta_{1}, \ldots, \eta_{N}\right]\right) .
\end{aligned}
$$

In the above definitions $T$ stands for transpose, I denotes an identity matrix of order $(N+1)$ and diag denotes a diagonal matrix of size $(N+1) \times(N+1)$. The boundary conditions (23) are imposed on the first and last rows of the matrices $\mathbf{C}$ and $\boldsymbol{\Phi}$ as follows 


$$
\left(\begin{array}{ccccc}
1 & 0 & \cdots & 0 & 0 \\
& & \mathbf{C} & & \\
0 & 0 & \cdots & 0 & 1
\end{array}\right)\left(\begin{array}{c}
\phi\left(\xi_{0}\right) \\
\phi\left(\xi_{1}\right) \\
\vdots \\
\phi\left(\xi_{N-1}\right) \\
\phi\left(\xi_{N}\right)
\end{array}\right)=\left(\begin{array}{c}
0 \\
0 \\
\vdots \\
0 \\
1
\end{array}\right)
$$

The solution for $\boldsymbol{\Phi}$ is then obtained from solving

$$
\boldsymbol{\Phi}=\mathbf{C}^{-1} \mathbf{K}_{2},
$$

where $\mathbf{K}_{2}$ is the right hand side of equation (27). Applying the Chebyshev spectral method on equation (13) to solve for $\theta(\eta)$ gives

$$
\mathbf{B \Theta}=-Q_{2} \Phi
$$

subject to the boundary conditions

$$
\theta\left(\xi_{N}\right)=1, \quad \theta\left(\xi_{0}\right)=0,
$$

where

$$
\begin{aligned}
\mathbf{B} & =\frac{1+R}{\operatorname{Pr}} \mathbf{D}^{2}+\left(2 \mathbf{j}+v_{0} \mathbf{I}\right) \mathbf{D}-\Omega \mathbf{I}, \\
\mathbf{\Theta}_{0} & =\left[\theta\left(\xi_{0}\right), \theta\left(\xi_{1}\right), \ldots, \theta\left(\xi_{N}\right)\right]^{T},
\end{aligned}
$$

The boundary conditions (30) are imposed on the first and last rows of the matrices in equation (29) as follows;

$$
\left(\begin{array}{ccccc}
1 & 0 & \cdots & 0 & 0 \\
& & & & \\
& & & & \\
0 & 0 & \cdots & 0 & 1
\end{array}\right)\left(\begin{array}{c}
\theta\left(\xi_{0}\right) \\
\theta\left(\xi_{1}\right) \\
\vdots \\
\theta\left(\xi_{N-1}\right) \\
\theta\left(\xi_{N}\right)
\end{array}\right)=\left(\begin{array}{c}
0 \\
-Q_{2} \phi\left(\xi_{1}\right) \\
\vdots \\
-Q_{2} \phi\left(\xi_{N-1}\right) \\
1
\end{array}\right)
$$

The solution for $\Theta$ is then obtained from solving

$$
\Theta=\mathbf{B}^{-1} \mathbf{K}_{1}
$$

where $\mathbf{K}_{1}$ is the right hand side of equation (34). Once the solutions for $\phi(\eta)$ and $\theta(\eta)$ have been found using equations (28) and (34), respectively, the SHAM approach is then used to solve then nonlinear equation (27) for $f(\eta)$.

In applying the SHAM, it is convenient to make the boundary conditions homogeneous by making use of the transformation

$$
F(\xi)=f(\eta)+f_{0}(\eta), \quad f_{0}(\eta)=e^{-\eta}
$$

where $f_{0}(\eta)$ is an initial guess chosen to satisfy the boundary conditions for $f(\eta)$. Substituting (35) in the governing equation (12) and the associated boundary conditions gives

$$
F^{\prime \prime}+a_{1}(\eta) F^{\prime}+a_{2}(\eta) F-\beta_{1} F^{2}=\psi(\eta),
$$


subject to

$$
F(0)=0, \quad F(\infty)=0,
$$

where

$$
\begin{aligned}
a_{1} & =2 \eta+v_{0}, \quad a_{2}=-\left(M+\frac{1}{D a}+\frac{2 R e F s f_{0}(\eta)}{D a}\right), \beta_{1}=\frac{R e F s}{D a}, \\
\psi & =-\left[f_{0}^{\prime \prime}+\left(2 \eta+v_{0}\right) f_{0}^{\prime}+G r \theta+G c \phi-M f_{0}-\frac{1}{D a} f_{0}-\frac{R e F s}{D a} f_{0}^{2}\right]
\end{aligned}
$$

We look for an initial approximation, $F_{0}(\eta)$, which is the solution of the linear part of the governing equation (36) given by

$$
F_{0}^{\prime \prime}+a_{1}(\eta) F_{0}^{\prime}+a_{2}(\eta) F_{0}=\psi(y)
$$

subject to

$$
F_{0}(0)=F_{0}(\infty)
$$

The problem (40) - (41) is first transformed, using (17), to the interval $[-1,1]$ then solved using the Chebyshev pseudospectral method. The unknown function $F_{0}(\xi)$ is approximated as

$$
F_{0}(\xi) \approx \sum_{k=0}^{N} F_{0}\left(\xi_{k}\right) T_{k}\left(\xi_{j}\right), \quad j=0,1, \ldots, N
$$

After transforming the derivatives and substituting equations (42) in (40) - (41) we obtain

$$
\mathrm{AF}_{0}=\Psi
$$

subject to the boundary conditions

$$
F_{0}\left(\xi_{N}\right)=F_{0}\left(\xi_{0}\right)=0,
$$

where

$$
\begin{aligned}
\mathbf{A} & =\mathbf{D}^{2}+\mathbf{a}_{1} \mathbf{D}+\mathbf{a}_{2}, \\
\mathbf{F}_{0} & =\left[F_{0}\left(\xi_{0}\right), F_{0}\left(\xi_{1}\right), \ldots, F_{0}\left(\xi_{N}\right)\right]^{T}, \\
\mathbf{\Psi} & =\left[\psi\left(\xi_{0}\right), \psi\left(\xi_{1}\right), \ldots, \phi\left(\xi_{N}\right)\right]^{T}, \\
\mathbf{a}_{s} & =\operatorname{diag}\left(\left[a_{S}\left(\eta_{0}\right), a_{S}\left(\eta_{1}\right), \ldots, a_{S}\left(\eta_{N-1}\right), a_{S}\left(\eta_{N}\right)\right]\right), \quad s=1,2,
\end{aligned}
$$

The boundary conditions (44) are imposed on the first and last rows of the matrices $\mathbf{A}$ and $\Psi$ as follows

$$
\left(\begin{array}{ccccc}
1 & 0 & \cdots & 0 & 0 \\
& & & & \\
& & & & \\
0 & 0 & \cdots & 0 & 1
\end{array}\right)\left(\begin{array}{c}
F_{0}\left(\xi_{0}\right) \\
F_{0}\left(\xi_{1}\right) \\
\vdots \\
F_{0}\left(\xi_{N-1}\right) \\
F_{0}\left(\xi_{N}\right)
\end{array}\right)=\left(\begin{array}{c}
0 \\
\psi\left(\xi_{1}\right) \\
\vdots \\
\psi\left(\xi_{N-1}\right) \\
0
\end{array}\right)
$$

The solution for $\mathbf{F}_{0}$ is then obtained from solving

$$
\mathbf{F}_{0}=\mathbf{A}^{-1} \mathbf{K}_{0}
$$


where $\mathbf{K}_{0}$ is the right hand side of (49).

To find the SHAM solutions of (36) we begin by defining the following linear operator

$$
\mathcal{L}[\tilde{F}(\eta ; q)]=\frac{\partial^{2} \tilde{F}}{\partial \eta^{2}}+a_{1} \frac{\partial \tilde{F}}{\partial \eta}+a_{2} \tilde{F}
$$

where $q \in[0,1]$ is the embedding parameter, and $\tilde{F}(\eta ; q)$ is an unknown function. The zeroth order deformation equation is given by

$$
(1-q) \mathcal{L}\left[\tilde{F}(\eta ; q)-F_{0}(\eta)\right]=q \hbar\{\mathcal{N}[\tilde{F}(\eta ; q)]-\psi(\eta)\}
$$

where $\hbar$ is the non-zero convergence controlling auxiliary parameter and $\mathcal{N}$ is a nonlinear operator given by

$$
\mathcal{N}[\tilde{F}(\eta ; q)]=\frac{\partial^{2} \tilde{F}}{\partial \eta^{2}}+a_{1} \frac{\partial \tilde{F}}{\partial \eta}+a_{2} \tilde{F}-\beta_{1} \tilde{F}^{2}
$$

Differentiating (52) $m$ times with respect to $q$ and then setting $q=0$ and finally dividing the resulting equations by $m$ ! yields the $m$ th order deformation equations

$$
\mathcal{L}\left[F_{m}(\eta)-\chi_{m} F_{m-1}(\eta)\right]=\hbar R_{m}(\eta),
$$

subject to the boundary conditions

$$
F_{m}(0)=F_{m}(\infty)
$$

where

$$
R_{m}(\eta)=F_{m-1}^{\prime \prime}+a_{1} F_{m-1}^{\prime}+a_{2} F_{m-1}-\beta_{1} \sum_{n=0}^{m-1} F_{n} F_{m-1-n}-\psi(y)\left(1-\chi_{m}\right)
$$

and

$$
\chi_{m}=\left\{\begin{array}{ll}
0, & m \leq 1 \\
1, & m>1
\end{array} .\right.
$$

Using the transformation (17) and applying the Chebyshev pseudospectral transformation on equations (54)-(56) gives

$$
\mathbf{A F}_{m}=\left(\chi_{m}+\hbar\right) \mathbf{A F}_{m-1}-\hbar\left(1-\chi_{m}\right) \mathbf{\Psi}+\hbar P_{m-1}
$$

subject to the boundary conditions

$$
F_{m}\left(\xi_{N}\right)=F_{m}\left(\xi_{0}\right)=0,
$$

where $A$ and $\boldsymbol{\Phi}$, are as defined in (45) and (47), respectively, and

$$
\begin{aligned}
\mathbf{F}_{m} & =\left[F_{m}\left(\xi_{0}\right), F_{m}\left(\xi_{1}\right), \ldots, F_{m}\left(\xi_{N}\right)\right]^{T}, \\
P_{m-1} & =-\beta_{1} \sum_{n=0}^{m-1} \mathbf{F}_{n} \mathbf{F}_{m-1-n}
\end{aligned}
$$

To implement the boundary conditions (59) we delete the first and last rows of $P_{m-1}$ and $\Psi$ and delete the first and last row and column of $\mathbf{A}$ This results in the following recursive formula for $m \geq 1$.

$$
\mathbf{F}_{m}=\left(\chi_{m}+\hbar\right) \mathbf{F}_{m-1}+\hbar \mathbf{A}^{-1}\left[\mathbf{P}_{m-1}-\left(1-\chi_{m}\right) \mathbf{\Psi}\right],
$$

Thus, starting from the initial approximation, which is obtained from (50), higher order approximations $F_{m}(\xi)$ for $m \geq 1$, can be obtained through the recursive formula (62). 


\section{Results and discussion}

In this section we give numerical results obtained by the spectral homotopy analysis method for the main parameters affecting the flow. Tables 1-6 display results for the skin friction $f^{\prime}(0)$, Nusselt number, $-\theta^{\prime}(0)$ and the Sherwood number $-\phi(0)$ when different parameters are varied. Table 1 gives the values of the local skin-friction for different values of the Hartmann number $M$. Analysis of the tabular data shows that magnetic field strength enhances the local skin friction as highlighted with increases in the absolute values of the skin friction as $M$ increases. Physically, this implies that the plate surface exerts a drag force on the fluid. Table 2 shows the effect of the local Darcy number on the local skin friction. From this table we observe that the skin friction decreases in absolute values as the Darcy parameter increases

\begin{tabular}{cccccc}
\hline$M$ & 2nd order & 4th order & 6th order & 8th order & 10th order \\
\hline 1 & -1.84655375 & -1.84671328 & -1.84671464 & -1.84671465 & -1.84671465 \\
5 & -2.78956864 & -2.78968689 & -2.78968758 & -2.78968758 & -2.78968758 \\
10 & -3.65197128 & -3.65204866 & -3.65204895 & -3.65204896 & -3.65204896 \\
15 & -4.34317940 & -4.34323240 & -4.34323255 & -4.34323255 & -4.34323255 \\
20 & -4.93446135 & -4.93449949 & -4.93449957 & -4.93449957 & -4.93449957 \\
\hline
\end{tabular}

Table 1. Values of the $f^{\prime}(0)$ for different values of $M$ at different SHAM orders

\begin{tabular}{cccccc}
\hline $\mathrm{D} a$ & 2nd order & 3rd order & 4th order & 6th order & 8th order \\
\hline 1 & -1.84655375 & -1.84670017 & -1.84671328 & -1.84671464 & -1.84671465 \\
5 & -1.43582267 & -1.43582436 & -1.43582440 & -1.43582440 & -1.43582440 \\
10 & -1.37843023 & -1.37843045 & -1.37843046 & -1.37843046 & -1.37843046 \\
15 & -1.35893671 & -1.35893678 & -1.35893678 & -1.35893678 & -1.35893678 \\
20 & -1.34911952 & -1.34911955 & -1.34911955 & -1.34911955 & -1.34911955 \\
\hline
\end{tabular}

Table 2. Values of the $f^{\prime}(0)$ for different values of $D a$ at different SHAM orders

The effect of thermal radiation on the skin friction is depicted on Table 3. We clearly observe in this table that the absolute values of the skin friction are reduced as the thermal radiation parameter $R$ increases. Table 4 has been prepared to show the effect of wall suction velocity on the skin friction. We observe that the magnitude of the local skin friction significantly increases with increasing values of the wall suction velocity.

\begin{tabular}{cccccc}
\hline$R$ & 2nd order & 4th order & 6th order & 8th order & 10th order \\
\hline 5 & -1.71772583 & -1.71778800 & -1.71778832 & -1.71778833 & -1.71778833 \\
10 & -1.64867944 & -1.64870680 & -1.64870690 & -1.64870690 & -1.64870690 \\
15 & -1.60822374 & -1.60823854 & -1.60823858 & -1.60823858 & -1.60823858 \\
20 & -1.58025980 & -1.58026899 & -1.58026901 & -1.58026901 & -1.58026901 \\
30 & -1.54255011 & -1.54255482 & -1.54255483 & -1.54255483 & -1.54255483 \\
\hline
\end{tabular}

Table 3. Values of the $f^{\prime}(0)$ for different values of $R$ at different SHAM orders

\begin{tabular}{cccccc}
\hline$v_{0}$ & 2nd order & 4th order & 6th order & 8th order & 10th order \\
\hline 1 & -1.84422963 & -1.84438345 & -1.84438474 & -1.84438475 & -1.84438475 \\
2 & -2.56784574 & -2.56822755 & -2.56823124 & -2.56823128 & -2.56823128 \\
3 & -3.38334080 & -3.38395995 & -3.38396583 & -3.38396590 & -3.38396590 \\
4 & -4.26060301 & -4.26139618 & -4.26140302 & -4.26140309 & -4.26140309 \\
5 & -5.17790146 & -5.17878811 & -5.17879476 & -5.17879482 & -5.17879482 \\
\hline
\end{tabular}

Table 4 . Values of the $f^{\prime}(0)$ for different values of $v_{0}$ at different SHAM orders 
Table 5 has been included to show the effects of varying the wall suction velocity $v_{0}$, thermal radiation parameter $R$, heat absorption parameter $\Omega$, absorption of radiation parameter $Q_{2}$ and the Prandtl number $\operatorname{Pr}$ on the local Nusselt number. It can be clearly observed that the rate of heat transfer between the wall and the fluid increases for increasing values of $v_{0}$, high values of $Q_{2}$ as well as $\operatorname{Pr}$. The Nusselt number is observed to be reduced by increasing values of $R$ and small values of $Q_{2}$.

\begin{tabular}{cccccc}
\hline$v_{0}$ & $R$ & $\Omega$ & $Q_{2}$ & $\operatorname{Pr}$ & $-\theta^{\prime}(0)$ \\
\hline 1.0 & 1.0 & 1.0 & 1.0 & 0.7 & 0.95840112 \\
2.0 & 1.0 & 1.0 & 1.0 & 0.7 & 1.22084437 \\
3.0 & 1.0 & 1.0 & 1.0 & 0.7 & 1.50119641 \\
4.0 & 1.0 & 1.0 & 1.0 & 0.7 & 1.79526126 \\
5.0 & 1.0 & 1.0 & 1.0 & 0.7 & 2.09985847 \\
\hline 1.0 & 2.0 & 1.0 & 1.0 & 0.7 & 0.76503772 \\
1.0 & 4.0 & 1.0 & 1.0 & 0.7 & 0.58038779 \\
1.0 & 5.0 & 1.0 & 1.0 & 0.7 & 0.52676177 \\
1.0 & 10.0 & 1.0 & 1.0 & 0.7 & 0.38340898 \\
\hline 1.0 & 1.0 & 2.0 & 1.0 & 0.7 & 1.12989889 \\
1.0 & 1.0 & 4.0 & 1.0 & 0.7 & 1.41719168 \\
1.0 & 1.0 & 5.0 & 1.0 & 0.7 & 1.54193396 \\
1.0 & 1.0 & 10.0 & 1.0 & 0.7 & 2.05653970 \\
\hline 1.0 & 1.0 & 1.0 & 2.0 & 0.7 & 0.81994800 \\
1.0 & 1.0 & 1.0 & 5.0 & 0.7 & 0.40458864 \\
1.0 & 1.0 & 1.0 & 10.0 & 0.7 & -0.28767696 \\
1.0 & 1.0 & 1.0 & 15.0 & 0.7 & -0.97994256 \\
1.0 & 1.0 & 1.0 & 20.0 & 0.7 & -1.67220816 \\
\hline 1.0 & 1.0 & 1.0 & 1.0 & 0.1 & 0.33842173 \\
1.0 & 1.0 & 1.0 & 1.0 & 0.7 & 0.95840112 \\
1.0 & 1.0 & 1.0 & 1.0 & 1.0 & 1.17519349 \\
1.0 & 1.0 & 1.0 & 1.0 & 10.0 & 5.39718685 \\
1.0 & 1.0 & 1.0 & 1.0 & 50.0 & 20.81046541 \\
\hline$\theta$
\end{tabular}

Table 5. Values of the $-\theta^{\prime}(0)$ for different values of $v_{0}, R, \Omega, Q_{2}$ and $P r$

Table 6 depicts the effects of varying $v_{0}, \gamma$ and $S c$ on the local Sherwood number. We observe from this table that all these three parameters cause the local Sherwood number to increase.

Final results are computed for the main physical parameters which are presented by means of graphs. The influence of the thermal Grashof number $G r$, the magnetic field parameter $M$, the Darcy number $D a$, absorption radiation parameter $Q_{2}$, thermal radiation parameter $R$, heat absorption $\Omega$, and the suction parameter $v_{0}$ on the velocity profiles can be analyzed from Figs. (a)-(g). Fig (a) shows the influence of thermal buoyancy force parameter $G r$ on the velocity. As can be seen from this figure, the velocity profile increases with increases in the values of the thermal buoyancy. We actually observe that the velocity overshoot in the boundary layer region. Buoyancy force acts like a favourable pressure gradient which accelerates the fluid within the boundary layer therefore the solutal buoyancy force parameter $G m$ has the same effect on the velocity as Gr. From Fig (b) we observe that the effect of magnetic field is to decrease the value of velocity profile through out the boundary layer which result in the thinning of the boundary layer thickness. Fig (c) displays the influence of the Darcy number $D a$ on the velocity profile. Increasing the Darcy number increases the velocity. The effect 
of increasing the value of the absorption parameter on the velocity is shown in Fig (d). We observe in this figure that increasing the value of the absorption of the radiation parameter due to increase in the buoyancy force accelerates the flow rate.

Fig (e) depicts the effect of varying thermal radiation parameter $R$ on the flow velocity. We observe that the thermal radiation enhances convective flow. Fig (f) illustrates the influence of heat absorption coefficient $\Omega$ on the velocity. Physically, the presence of heat absorption (thermal sink) effect has the tendency in resulting in a net reduction in the flow velocity. This behaviour is seen from Fig (f) in which the velocity decreases as $\Omega$ increases. The hydrodynamic boundary layer decreases as the heat absorption effects increase. The effects of $v_{0}$ on the velocity field are shown in Fig (g). It is clearly seen from this figure that the velocity profiles decrease monotonically with the increase of suction parameter indicating the usual fact that suction stabilizes the boundary layer growth.

The influence of heat absorption, radiation absorption, thermal radiation and suction on the temperature distribution is respectively, shown on Figs (h) - (k). Fig (h) depicts the effects of heat absorption $\Omega$ on the temperature distribution. It is observed that the boundary layer absorbs energy resulting in the temperature to fall considerably with increasing values of $\Omega$. This is because when heat is absorbed, the buoyancy force decreases the temperature profile. The effect of absorbtion of radiation parameter on the temperature profile is shown on Fig (i). It is seen from this figure that the effect of absorption of radiation is to increase temperature in the boundary layer as the radiated heat is absorbed by the fluid which in turn increases the temperature of the fluid very close to the porous boundary layer and its effect diminishes far away from the boundary layer. From Fig (j) we observe that the effect of thermal radiation is to enhance heat transfer as thermal boundary layer thickness increases with increase in the thermal radiation. We observe that the effect of $R$ is to increase the temperature distribution in the thermal boundary layer. This is because the increase of $R$ implies increasing of radiation in the thermal boundary layer, and hence increases the values of the temperature profiles in thermal boundary layer. Lastly the effect of suction parameter $v_{0}$ on the temperature field is displayed in Fig (k). We see that the temperature profiles decrease with increasing values of $v_{0}$. This is because sucking decelerates fluid particles through the porous wall reducing the growth of the fluid boundary layer as well as thermal and concentration boundary layers.

\begin{tabular}{cccc}
\hline$v_{0}$ & $\gamma$ & $S c$ & $-\phi^{\prime}(0)$ \\
\hline 1 & 1 & 0.6 & 1.53261796 \\
2 & 1 & 0.6 & 1.97076626 \\
3 & 1 & 0.6 & 2.44776350 \\
4 & 1 & 0.6 & 2.95336689 \\
5 & 1 & 0.6 & 3.47988849 \\
\hline 1 & 0 & 0.6 & 1.28843084 \\
1 & 5 & 0.6 & 2.25555303 \\
1 & 10 & 0.6 & 2.90467826 \\
1 & 20 & 0.6 & 3.87130625 \\
1 & 50 & 0.6 & 5.84331065 \\
\hline 1 & 1 & 0.6 & 1.53261796 \\
1 & 1 & 1.0 & 2.13711784 \\
1 & 1 & 2.0 & 3.45301022 \\
1 & 1 & 5.0 & 6.90551441 \\
1 & 1 & 10.0 & 12.23149717 \\
\hline
\end{tabular}

Table 6. Values of the $-\phi^{\prime}(0)$ for different values of $v_{0}, \gamma$ and $S c$ 


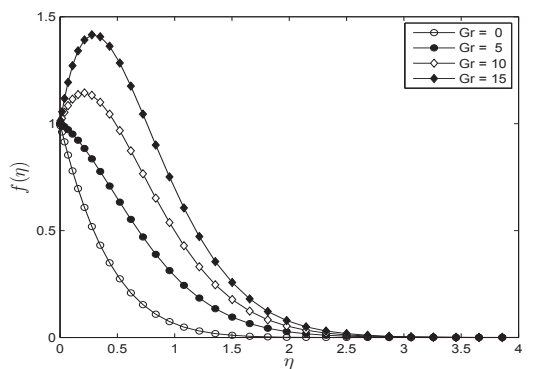

(a) Plot of $f^{\prime}(\eta)$ for varying $G r$

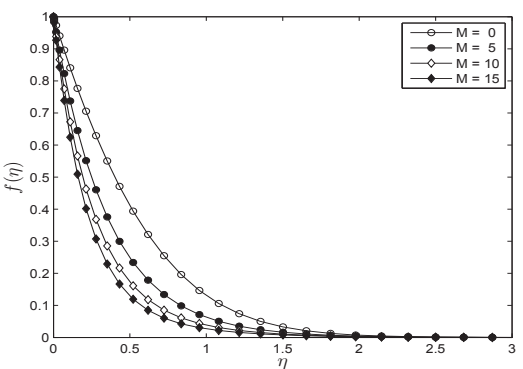

(b) Plot of $f^{\prime}(\eta)$ for varying $M$

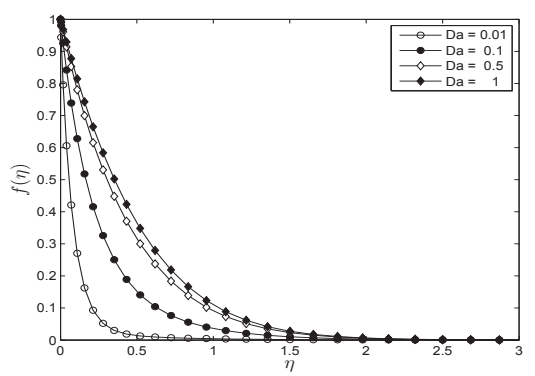

(c) Plot of $f^{\prime}(\eta)$ for varying $D a$

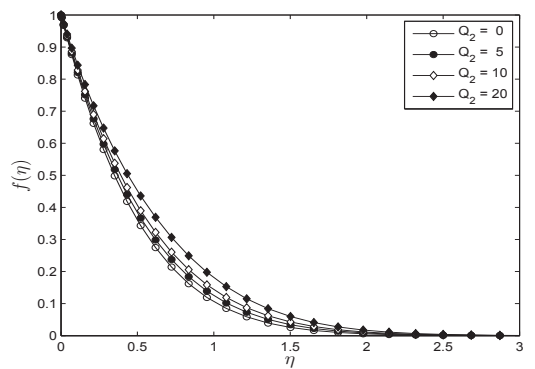

(d) Plot of $f(\eta)$ for varying $Q_{2}$

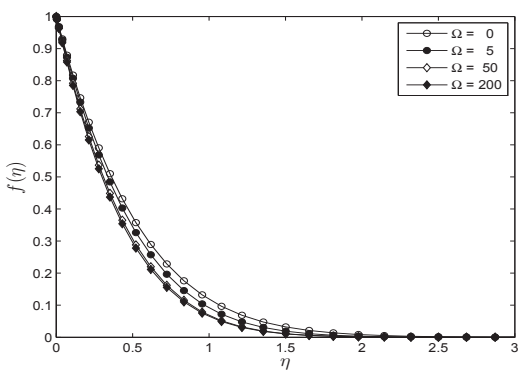

(f) Plot of $f(\eta)$ for varying $\Omega$

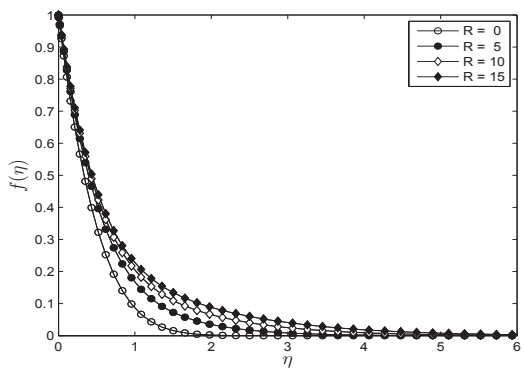

(e) Plot of $f(\eta)$ for varying $R$

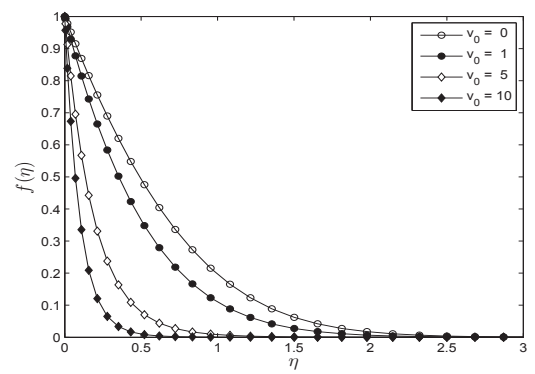

(g) Plot of $f(\eta)$ for varying $v_{0}$ 


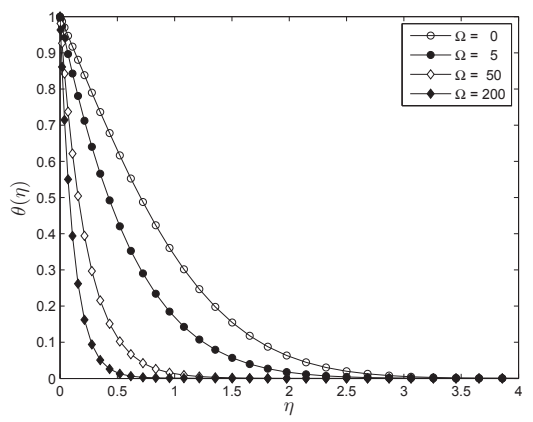

(h) Plot of $\theta(\eta)$ for varying $\Omega$

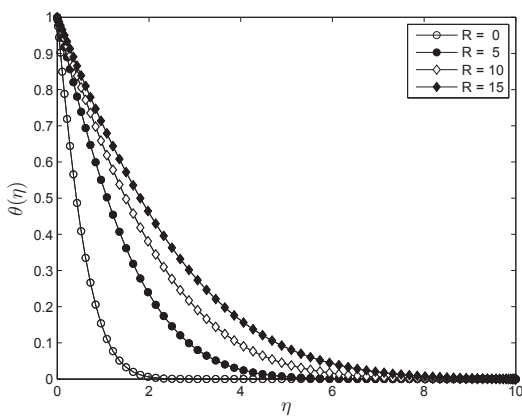

(j) Plot of $\theta(\eta)$ for varying $R$

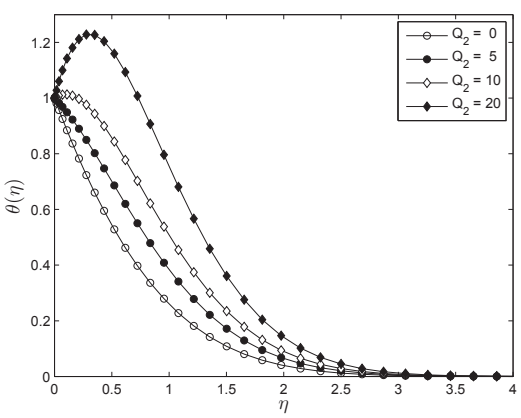

(i) Plot of $\theta(\eta)$ for varying $Q_{2}$

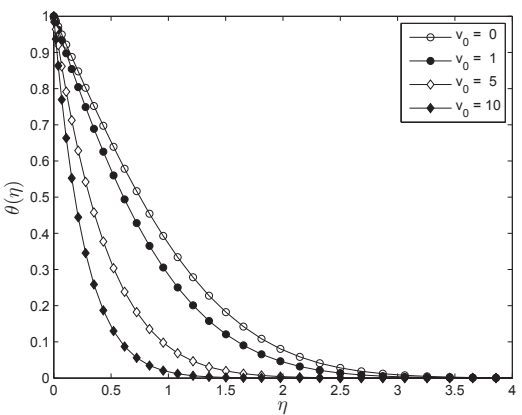

(k) Plot of $\theta(\eta)$ for varying $v_{0}$

Figs (l) and (m) depict the influence of the non-dimensional chemical reaction parameter $\gamma$ and the suction parameter on concentration profiles, respectively. The effect of chemical reaction parameter is very important in the concentration field. Chemical reaction increases the rate of interfacial mass transfer. Reaction reduces the local concentration, thus increases its concentration gradient and its flux. In Fig (m) we see that the concentration profiles decrease with increasing values of the suction parameter. 


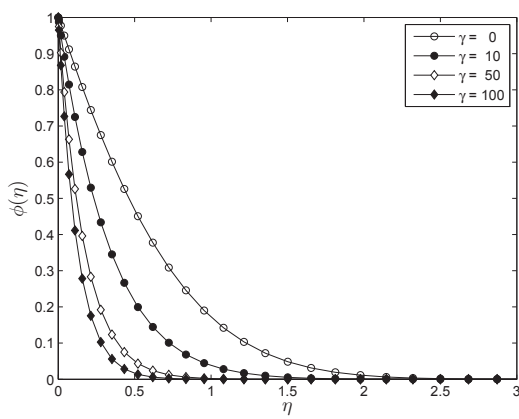

(l) Plot of $\phi(\eta)$ for varying $\gamma$

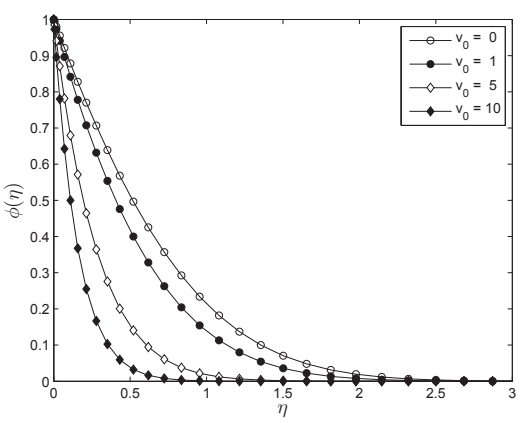

(m) Plot of $\phi(\eta)$ for varying $v_{0}$

\section{Conclusions}

In this chapter, a new improved numerical technique to solve the problem of unsteady magnetohydrodynamic convective heat and mass transfer past an infinite permeable vertical plate in porous medium with thermal radiation, heat absorption and chemical reaction has been employed. The highly non-linear momentum, energy and species boundary layer equations are converted into ordinary differential equations using similarity transformations before being solved using the spectral homotopy analysis method. The effects of various physical parameters like buoyancy parameter, Hartmann number, Darcy number, suction, thermal radiation and chemical reaction on velocity, temperature and concentration profiles are obtained.

The following main conclusions can be drawn from the present chapter:

1. Wall suction stabilizes the velocity, thermal as well as concentration boundary layer growth.

2. Boundary layer flow attain minimum velocity values for large Hartmann numbers.

3. Buoyancy parameter is to increase the velocity distribution in the momentum boundary layer.

4. The presence of heat absorption effects cause reductions in the fluid temperature which resulted in decreases in the fluid velocity.

5. The concentration decreases with increasing the chemical reaction parameter.

6. Both the velocity and temperature profiles increase with increasing values of radiation absorption parameter.

These results might find wide applications in engineering, such as geothermal system, heat exchangers, and nuclear waste depositors. 


\section{References}

[1] Cussler, E.L. (1988). Diffusion Mass Transfer in Fluid Systems, Cambridge University Press, London.

[2] Das, U.N, Deka, R., \& Soundalgekar, V.M. (1994). Effects of mass transfer on flow past an impulsively started infinite vertical plate with constant heat flux and chemical reaction. J. Forshung Im Ingenieurwesen-Engineering Research Bd, 60, 284-287.

[3] Anjalidevi, S.P. \& Kandasamy, R. (1999). Effects of chemical reaction, heat and mass transfer on laminar flow along a semi infinite horizontal plate. Heat and Mass Transfer, 35, 465-467.

[4] Seddeek, M.A., Darwish, A.A. \& Abdelmeguid, M.S. (2007). Effects of chemical reaction and variable viscosity on hydromagnetic mixed convection heat and mass transfer for Hiemenz flow through porous media with radiation, Commun Nonlinear Sci. Numer. simulat., 15, 195-213.

[5] Salem, A.M. \& Abd El-Aziz, M. (2008). Effect of Hall currents and chemical reaction on hydromagnetic flow of a stretching vertical surface with internal heat generation/absorption. Applied Mathematical Modelling, 32, 1236-1254.

[6] Mohamed, R. A., (2009). Double-Diffusive Convection-radiation Interaction on Unsteady MHD Flow over a Vertical Moving Porous Plate with Heat Generation and Soret Effects, Applied Mathematical Sciences, 3, (13), 629-651.

[7] Ibrahim, F.S., Elaiw, A.M., \& Bakr, A.A. (2008). Effect of chemical reaction and radiation absorption on the unsteady MHD free convection flow past a semi infinite vertical permeable moving plate with heat source and suction. Commun Nonlinear Sci. Numer. simulat., 13, 1056-1066.

[8] Mahdy, A. (2010). Effect of chemical reaction and heat generation or absorption on double-diffusive convection from a vertical truncated cone in a porous media with variable viscosity. International Communications in Heat and Mass Transfer, 37, 548-554.

[9] Nield, D. A., \& Bejan, A., (1999). Convection in Porous Media, 2nd edition. Springer-Verlag, New York .

[10] Abel, M.S., Kan, S.K., Prasad, K.V. (2001). Convective heat and mass transfer in a visco-elastic fluid flow through a porous medium over a stretching sheet. International Journal of Numerical Methods for Heat and Fluid flow, 11(8), 779-792.

[11] Ali, M.E. (2007). The effect of lateral mass flux on the natural convection boundary layers induced by a heated vertical plate embedded in a saturated porous medium with internal heat generation. Int. J. Thermal Sci., 46, 157-163.

[12] Vajravelu, K., \& Hadjinicolaou, A., (1997). Convective heat transfer in an electrically conducting fluid at a stretching surface with uniform free stream.Int. J. Eng. Sci, 35, 1237-1244.

[13] Chamkha, A. J., \& Khaled, A. R. A., (2000). Similarity solutions for hydromagnetic mixed convection heat and mass transfer for Hiemenz flow through a porous medium. Int.J.Numerical Methods for Heat and Fluid Flow, 10, 94-115.

[14] Shateyi, S., Sibanda, P., \& Motsa, S.S., (2007). Magnetohydrodynamic flow past a vertical plate with radiative heat transfer, Journal of Heat Transfer, 129, 1708-1714.

[15] Shateyi, S. Motsa, S.S., \& Sibanda, P. (2010). The Effects of Thermal Radiation, Hall Currents, Soret, and Dufour on MHD Flow by Mixed Convection over a Vertical Surface in Porous Media, Mathematical Problems in Engineering, Volume 2010, Article ID 627475, 20 pages doi:10.1155/2010/627475.

[16] Makinde, O.D \& Sibanda, P. (2008). Magnetohydrodynamic mixed convective flow and 
heat and mass transfer past a vertical plate in a porous medium with constant wall suction. ASME - Journal of Heat Transfer , 130 (11260), 1-8.

[17] Bég, O.A., Zueco, J., Bhargava, R., \& Takhar, H.S. (2009). Magnetohydrodynamic convection flow from a sphere to a non-Darcian porous medium with heat generation or absorption effects: network simulation. International Journal of Thermal Sciences, 48, 913-921.

[18] Pal, A. \& Talukdar, B. (2010). Perturbation analysis of unsteady magnetohydrodynamic convective heat and mass transfer in a boundary layer slip flow past a vertical permeable plate with a thermal radiation and chemical reaction, Commun Nonlinear Sci. Numer. simulat., 15, 1813-1830.

[19] Makinde, O.D \& Aziz, A. (2010). MHD mixed convection from a vertical plate embedded in a porous medium with a convective boundary condition. International Journal of Thermal Sciences, 49, 1813-1820.

[20] Crepeau, J.C. \& Clarksean, R. (1997). Similarity solutions of natural convection with internal heat generation J. Heat Transfer, 119, 183-185.

[21] Patil P.M., \& Kulkarni, P.S. (2008). Effects of chemical reaction on free convective flow of a polar fluid through a porous medium in the presence of internal heat generation. International Journal of Thermal Sciences, 47, 1043-1054.

[22] Salem, M.A., \& Abd El-Aziz, M. (2008). Effect of Hall currents and chemical reaction on hydromagnetic flow of a stretching vertical surface with internal heat generation/absorption. Applied Mathematical Modelling, 32, 1236-1254.

[23] Samad, M.A., \& Mohebujjaman, M. (2009). MHD Heat and Mass Transfer Free Convection Flow anong a Vertical Stretching Sheet in Presence of Magnetic Field with Heat Generation. Research Journal of Applied Science, Engineering and Technology, 1(3), 98-106.

[24] Siegel, R., \& Howell, J.R. (2001). Thermal Radiation Heat Transfer, Speedy Hen, London, CA, United Kingdom, (ISBN: 1560328398 / 1-56032-839-8).

[25] C. Canuto, M. Y. Hussaini, A. Quarteroni, and T. A. Zang (1998). Spectral Methods in Fluid Dynamics, Springer-Verlag, Berlin, 1988.

[26] Cortell, R., (2008). Effects of viscous dissipation and radiation on the thermal boundary layer over a nonlinearly stretching sheet. Phys Lett A, 372, 631-636.

[27] Bataller, R.C., (2008). Radiation effects for the Blasius and Sakiadis flows with a convective surface boundary condition. Appl Math Comput, 206: 832-840.

[28] Ibrahim, F.S., Elaiw, A.M., \& Bakar, A.A. (2008). Effect of the chemical reaction and radiation absorption on the unsteady MHD free convection flow past a semi infinite vertical permeable moving plate with heat source and suction. Communications in Nonlinear Science and Numerical Simulation, 13, 1056-1066.

[29] Shateyi, S. (2008). Thermal Radiation and Buoyancy Effects on Heat and Mass Transfer over a Semi-Infinite Stretching Surface with Suction and Blowing, Journal of Applied Mathematics, Volume 2008, Article ID 414830, 12 pages doi:10.1155/2008/414830.

[30] Shateyi, S., \& Motsa, S.S. (2009). Thermal Radiation Effects on Heat and Mass Transfer over an Unsteady Stretching Surface. Mathematical Problems in Engineering, Volume 2009, Article ID 965603, 13 pages doi:10.1155/2009/965603.

[31] Aliakbar, V., Alizadeh-Pahlavan, A. \& Sadeghy, K., (2009). The influence of thermal radiation on MHD flow of Maxwellian fluids above stretching sheets. Commun Nonlinear Sci Numer Simulat, 14(3): 779-794.

[32] Hayat, T., \& Qasim, M. (2010). Influence of thermal radiation and Joule heating on MHD 
flow of a Maxwell fluid in the presence of thermophoresis. Int. J. Heat Mass Transfer, doi:10.1016/j.ijheatmasstransfer.2010.06.014.

[33] Cortell, R, (2010). Suction, viscous dissipation and thermal radiation effects on the flow and heat transfer of a power-law fluid past an infinite porous plate, Chem Eng Res Des, doi:10.1016/j.cherd.2010.04.017

[34] S.J. Liao (2003), Beyond perturbation: Introduction to homotopy analysis method. Chapman \& Hall/CRC Press, 2003.

[35] S. S. Motsa, P. Sibanda and S. Shateyi (2010a), A new spectral-homotopy analysis method for solving a nonlinear second order BVP. Commun. Nonlinear Sci. Numer. Simulat. 15 (2010a) 2293-2302.

[36] S. S. Motsa, P. Sibanda, F. G. Awad, S. Shateyi (2010b), A new spectral-homotopy analysis method for the MHD Jeffery-Hamel problem, Computer \& Fluids , 39 (2010b) 1219-1225.

[37] M. A. Hossain, M.A. Alim, and D. A. S. Rees (1999), The effect of radiation on free convection from a porous vertical plate. Int. J. Heat Mass Transfer, 42, (1999), 181 - 191.

[38] A. Raptis (1998), Flow of a micropolar fluid past a continuously moving plate by the presence of radiation, Int. J. Heat Mass Transfer, 41, (1998), 2865-2866.

[39] L. N. Trefethen (2000), Spectral Methods in MATLAB, SIAM, 2000. 


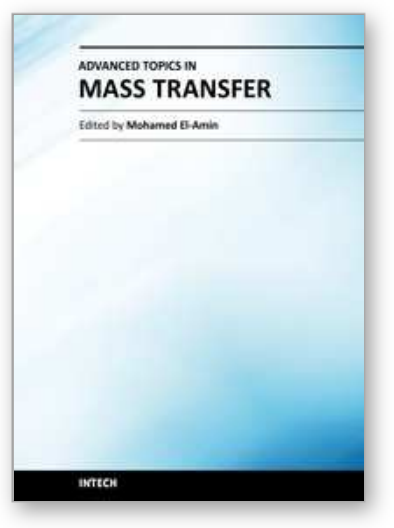

\author{
Advanced Topics in Mass Transfer \\ Edited by Prof. Mohamed El-Amin
}

ISBN 978-953-307-333-0

Hard cover, 626 pages

Publisher InTech

Published online 21, February, 2011

Published in print edition February, 2011

This book introduces a number of selected advanced topics in mass transfer phenomenon and covers its theoretical, numerical, modeling and experimental aspects. The 26 chapters of this book are divided into five parts. The first is devoted to the study of some problems of mass transfer in microchannels, turbulence, waves and plasma, while chapters regarding mass transfer with hydro-, magnetohydro- and electro- dynamics are collected in the second part. The third part deals with mass transfer in food, such as rice, cheese, fruits and vegetables, and the fourth focuses on mass transfer in some large-scale applications such as geomorphologic studies. The last part introduces several issues of combined heat and mass transfer phenomena. The book can be considered as a rich reference for researchers and engineers working in the field of mass transfer and its related topics.

\title{
How to reference
}

In order to correctly reference this scholarly work, feel free to copy and paste the following:

Stanford Shateyi and Sandile Motsa (2011). Unsteady Magnetohydrodynamic Convective Heat and Mass Transfer Past an Infinite Vertical Plate in a Porous Medium with Thermal Radiation, Heat Generation/Absorption and Chemical Reaction, Advanced Topics in Mass Transfer, Prof. Mohamed El-Amin (Ed.), ISBN: 978-953-307-333-0, InTech, Available from: http://www.intechopen.com/books/advanced-topicsin-mass-transfer/unsteady-magnetohydrodynamic-convective-heat-and-mass-transfer-past-an-infinite-verticalplate-in-a-

\section{INTECH}

open science | open minds

\section{InTech Europe}

University Campus STeP Ri

Slavka Krautzeka 83/A

51000 Rijeka, Croatia

Phone: +385 (51) 770447

Fax: +385 (51) 686166

www.intechopen.com

\section{InTech China}

Unit 405, Office Block, Hotel Equatorial Shanghai

No.65, Yan An Road (West), Shanghai, 200040, China

中国上海市延安西路 65 号上海国际贵都大饭店办公楼 405 单元

Phone: +86-21-62489820

Fax: $+86-21-62489821$ 
(C) 2011 The Author(s). Licensee IntechOpen. This chapter is distributed under the terms of the Creative Commons Attribution-NonCommercialShareAlike-3.0 License, which permits use, distribution and reproduction for non-commercial purposes, provided the original is properly cited and derivative works building on this content are distributed under the same license. 